rev.relac.int.estrateg.segur.8(1):21-44,2013

\title{
CONSENSO Y POS-CONSENSO DE WASHINGTON: DOS GENERACIONES DE REFORMAS INCOMPLETAS*
}

\author{
Héctor Cuadra Montiel ${ }^{* *}$
}

\section{RESUMEN}

La dimensión perdida de las reformas emprendidas tanto por el énfasis macro-económico del Consenso de Washington, como por el tenor institucional del pos-Consenso radica en lo político y lo social. La pretensión de armonización de ambas generaciones de reformas ha fallado en reconocer la centralidad política de los actores en los procesos en los que toman parte activa, ignorando el carácter intencional, contingente e impredecible que logran imprimirle.

Palabras clave: Consenso, pos-Consenso, reforma, economía, institución, actor, agencia

* El presente artículo es una versión revisada, ampliada y modificada del artículo de mi autoría publicado en el 2007, titulado Incompleteness of Post-Washington Consensus: A Critique of Macro-economic and Institutional Reforms, en International Studies, 44 (2), 103-122.

Agradezco el apoyo brindado por Nereida Castañón Soriano. Su asistencia en diversas tareas de investigación, edición y traducción resultaron de gran ayuda.

** Investigador del Programa Estudios Políticos e Internacionales, El Colegio de San Luis A.C., México. h.cuadra.montiele@gmail.com y hcuadra@colsan.edu.mx 


\title{
WASHINGTON CONSENSUS AND POST-CONSENSUS: TWO GENERATIONS OF INCOMPLETE REFORMS
}

\begin{abstract}
There is a missing dimension on the economic reforms of the washington consensus, as well as in the institutional turn of the post-washington consensus: the political and social aspects. policy harmonization of both generations of reforms has failed to acknowledge the political centrality of the actors (agencies) on those processes in which they are active, ignoring the intentional, contingent and unpredictable character they manage to give it.
\end{abstract}

Key words: consensus, post-consensus, reform, economy, institution, actor, agency

\section{CONSENSO E PÓS-CONSENSO DE WASHINGTON: DUAS GERAÇÕES DE REFORMAS INCOMPLETAS}

\section{RESUMO}

A dimensão perdida das reformas empreendidas tanto pela ênfase macro-econômica do Consenso de Washington, como pelo teor institucional do pós-Consenso, radica nas dimensões políticas e sociais. O objetivo de harmonizar as duas gerações de reformas falhou em reconhecer a centralidade política dos atores nos processos nos quais têm parte ativa e ignora a natureza intencional, contingente e imprevisível que consegue imprimir-lhe.

Palavras-chave: Consenso, pós- Consenso, reforma, economia, instituição, ator

\section{INTRODUCCIÓN}

Los procesos asociados con la supuestamente inevitable globalización no son impulsados por el mercado, sino que existe una dimensión social en dicha compulsión económica. Ni el discurso de la globalización ni el modelo de equilibrio general reconocen las diferencias institucionales y los determinantes sociales de los mercados, ya que se presenta como homogéneo y convergente.

La insuficiencia de la primera generación de reformas económicas asociadas con el Consenso de Washington y también de la importancia institucional de la segunda generación de reformas del pos-Consenso de Washington puso de relieve dimensiones sociales y políticas. A pesar de que sus énfasis varían y se complementan entre sí, ningún conjunto de reformas reconoce el carácter político vital de los actores y la acción social situada en su contexto, dado que las dos generaciones de reformas están orientadas hacia la armonización de las políticas. 
Al discutir sobre los límites del Consenso de Washington y el pos-Consenso de Washington, este artículo hace hincapié en la acción social contextualizada, con el fin de subrayar la importancia de los actores en los procesos sociales, políticos y económicos, ya que dialécticamente influyen a la vez que son influenciados por las condiciones que los rodean. Además de las circunstancias materiales, el papel de las ideas también se destaca en momentos históricos y límites geográficos específicos. Al rastrear las diferentes trayectorias de las reformas, que a su vez son concurrentes con tendencias y contratendencias en juego, demuestra ser beneficioso para destacar la dinámica de arraigo y pertenencia social, donde las interacciones de poder contribuyen a la contingencia de los sistemas abiertos (Hay, 1996, 2002; Jessop, 1990). Filosóficamente consecuente, teóricamente informado y empíricamente fundamentado se afirma que permiten destacar la importancia de las relaciones que no son susceptibles de observación directa. En este artículo se pretende analizar el contexto internacional estratégicamente selectivo de elementos materiales e ideacionales. Pero ello nunca determina el ritmo, el alcance y la profundidad de la transformación contemporánea social, económica y política en algunos países de América Latina. Claramente dichas dimensiones del contexto internacional no pueden ser ignoradas y deben ser cuidadosamente examinadas.

Como una manera de afrontar la incertidumbre de los procesos sociales de cambio, ha habido un énfasis en las reformas institucionales para complementar el desarrollo económico y la reestructuración de los países en desarrollo. Sin embargo, la deficiencia del pos-Consenso de Washington radica en su falta de atención a la función esencial que desempeñan los actores en la acción social, su recomendación de una idea determinista de convergencia y la suposición del abstracto equilibrio económico para lograr un crecimiento con equidad. Es difícil creer que esto suceda, ya que la segunda generación de reformas supone que fue equitativo el crecimiento, cosa que aún no se ha alcanzado. Ello es debido al incompleto carácter social del ajuste económico realizado.

\section{LA GLOBALIZACIÓN Y EL ESPEJISMO DE UNIDAD DE MERCADO}

Contrario a afirmaciones hiperglobalistas, la globalización no es un proceso estructurado y una condición ahistórica contra el cual no hay alternativas que se puedan ofrecer (Hay y Marsh, 2000; Held et al. 1999; Ohmae, 1990, 1996; Strange, 1994, 1996). En cuanto a su presunción de inevitable, Watson sugiere por qué el discurso político de la globalización a menudo se presenta como una fuerza económica impulsada con homogeneización. Centrándose en la teoría económica ortodoxa neo-clásica, el modelo de equilibrio general puede ser considerado como un precursor de la hipótesis de la globalización según la cual los mercados se ven afectados por las diferencias institucionales y los determinantes sociales, ya que están estrechamente relacionados a través del tiempo y el espacio (Watson, 2003). Sin darse cuenta que los supuestos de estos modelos de análisis no son realistas, y el hecho de que el equilibrio es sólo una propiedad de lograrse en el modelo, se ha utilizado de manera ideológica la prescripción de los cursos de política económica. Haciendo a un lado la jerga económica y los tecnicismos del modelo, la reconstrucción de un intercambio puro en condiciones de competencia perfecta se invoca en el 
simple y engañoso retrato de la globalización, donde el establecimiento de marcos populares de referencia se han extendido más allá de la academia (Watson, 2003).

El problema con la imposibilidad de aplicar el modelo de equilibrio general es la falta de coincidencia con la realidad, ya que la mayoría de la ortodoxia orientada matemáticamente en los modelos neoclásicos se basan en suposiciones abstractas, una ontología positivista e inferencias deductivistas (Lawson, 1997 y 2003; Cuadra-Montiel, 2007). Baste decir que la ontología positivista es propensa a un análisis determinista de la ciencia social a causa de la imposibilidad de realizar experimentación en un sistema cerrado. A diferencia de las ciencias naturales, las ciencias sociales se caracterizan por su falta de control experimental y la inexactitud de sus hallazgos. Igualmente problemático es el hecho de que el deductivismo hace inferencias a partir de lo general hacia las leyes particulares; la formulación en términos de conjeturas constantes son simplemente considerados como de validez universal. Por otra parte, otro tema crítico con los modelos de equilibrio en particular, y el modelado matemático en general, es que son incompatibles con un enfoque que considera la intención de los actores. Se considera la hipótesis de que sólo hay un curso racional de acción y, por tanto, solo una 'elección racional', lo cual niega la capacidad de un actor individual o colectivo para decidir sobre un curso de acción alternativo. Por la importancia en función de la intención de los actores, la indeterminación surge de los procesos, pero esto a su vez es el único mecanismo que garantiza que la condiciones de equilibrio no se violen (Lawson, 1997, 2003).

Siguiendo a Polanyi (1957), se argumenta que las actividades económicas forman parte de una más amplia red de relaciones sociales, y que no son reducibles a las operaciones de intercambio. Producción y la distribución son también componentes claves de los procesos económicos. Por otra parte, la mercantilización artificial de la mano de obra, del dinero, de la tierra y del conocimiento, constituyen contextos que tienen sesgos hacia ciertas políticas orientadas a resultados. Esta mercantilización ha dado forma a muchas formulaciones económicas contemporáneas, a saber, la idea de que el dinero debe ser suministrado por los mecanismos de ajuste automático, la creación de los mercados laborales y la promoción del libre comercioº . Tal política económica ha sido enérgicamente defendida por economistas neo-clásicos ortodoxos, quienes haciendo hincapié en los intercambios de mercado, han prestado poca atención a los problemas de la producción y la redistribución ${ }^{2}$.

1. Polanyi no menciona el conocimiento como una mercancía ficticia. Para él, la mercantilización tiene lugar cuando el trabajo del hombre y la explotación de la naturaleza es considerado como disponibles para la venta, y cuando la tierra tiene como objetivo obtener una renta de ella. Del mismo modo, el mercado de trabajo produce beneficios en la forma del salario, y el interés se considera la ganancia que se puede obtener de los mercados de dinero (Polanyi, 1957; Cuadra-Montiel, 2011).

2. Partiendo de una perspectiva antropológica, Polanyi sostiene que hay aspectos importantes en una economía que históricamente ha sido organizada, tales como la reciprocidad, la redistribución, y el intercambio. Para algunas sociedades antiguas dichas prácticas se organizaron para garantizar el orden en la producción y distribución (Polanyi, 1992, 1996). 
Debido a que todas las actividades económicas tienen un contexto social, son acciones socialmente situadas. Por lo tanto, las reglas, normas e instituciones de todas las clases son básicamente construcciones sociales (Granovetter, 1985, 1992, Granovetter y Swedberg, 1992; Swedberg, 1991, 1994). Visto desde este ángulo ideacional, los intercambios en el mercado también se pueden considerar como construcciones sociales que tienden a reproducir la idea originalmente sugerida por Adam Smith de que es la propensión de los hombres al trueque, intercambio y comercio de una cosa por otra (Smelser y Swedberg, 1994; Swedberg, 1994). A pesar de la ausencia del ánimo de lucro, esas suposiciones han sentado las bases para el énfasis contemporáneo en las relaciones de intercambio. Los economistas neoclásicos ortodoxos han sostenido que estas relaciones son pre-existentes y, por tanto, no están abiertas a discusión. Se parte de esos supuestos para considerar que la propensión al intercambio que alimentan el espejismo de la globalización es principalmente impulsada por el mercado. Tal constructo social es un material que ha dado forma al mundo en que vivimos.

\section{MULTILATERALISMO Y REGIONALISMO}

En ninguna parte son los supuestos sobre las relaciones de intercambio el motor del proceso social más evidente que en las obras de los economistas neoclásicos sobre comercio internacional e integración regional. Desde que Viner escribió acerca de la desviación del comercio y la creación de comercio en la década de 1950, el debate ha evolucionado a través de las diferentes fases (Bhagwati, 1999; Bhagwati, et al., 1998).

La orientación actual hacia criterios de convergencia y su empuje para la armonización representa un enfoque que contrasta con la Doctrina Calvo preferida por los latinoamericanos hace algunas décadas ${ }^{3}$. Sin embargo, los marcos regulatorios y las doctrinas a un lado, hacen evidente que la inversión extranjera directa (IED) se concentre sobre todo en las economías más desarrolladas del mundo, que se agrupan en tres regiones principales: en Europa Occidental los miembros de la Unión Europea, en América del Norte, especialmente los Estados Unidos, y en Asia Oriental, principalmente en Japón y China. Aunque ha habido un aumento en el volumen, el valor y la frecuencia tanto de salidas y de entradas específicas de IED entre las economías en

3. Diplomático argentino quien argumentó que la jurisdicción internacional en diferencias relativas a inversiones recae en el país en el que se encuentra la inversión. Por lo tanto, el inversor no tiene más remedio que recurrir a los tribunales y recursos locales, y debe renunciar a la protección diplomática de sus países de origen. Pretendió proporcionar una protección legal contra las regulaciones extraterritoriales de otros países que tienden a no atender las leyes de acogida y normas nacionales. La Doctrina Calvo prohíbe el uso de la intervención diplomática como medio de ejecución de las reclamaciones privadas ante recursos internos que se han agotado. Más limitado que la Doctrina Calvo, de la que creció, la Doctrina Drago, formulado por otro argentino, sostuvo que la deuda pública no debe ser recuperada por un Estado soberano mediante sus fuerzas armadas o a través de la ocupación de su territorio por un país extranjero. A partir de la década de 1980 países de América Latina han aceptado el arbitraje internacional para resolver controversias entre inversionistas y los gobiernos. 
desarrollo, la mayor proporción sigue siendo claramente concentrada en la tríada (Mansfield y Milner, 1997, 1999).

El espejismo de la inevitabilidad del mercado se manifiesta en diversas formas. Ya sean regionales o preferenciales los acuerdos comerciales constituyen bloques que ayudan u obstaculizan la creación de un orden multilateral de comercio. El comercio universal, libre y abierto está destinado a ser el objetivo final, no sólo para los gobiernos, sino también para la sociedad internacional. En términos estrictamente normativos, la proliferación de acuerdos comerciales desde la década de 1980 se percibe como intrínsecamente discriminatoria, ya que se teme que opere en detrimento de una verdadera liberalización multilateral y, por tanto, pueda fragmentar el sistema mundial de comercio (Bhagwati y Panagariya, 1999; Panagariya, 1999). Sin embargo, es posible ver que tal resultado es muy poco probable. Es necesario tener en cuenta diferentes niveles de negociación, es decir, los regionales y multilaterales no son, estrictamente hablando, opuestos uno al otro como la Organización Mundial de Comercio (OMC) evidencia particularmente en el Acuerdo General sobre Aranceles Aduaneros y Comercio (GATT) y el Acuerdo General sobre el Comercio de Servicios (AGCS). Para la Ronda Doha de negociaciones multilaterales es una cuestión importante a tener en cuenta. La cláusula de la nación más favorecida (NMF) no debe confundirse con el universalismo, dado que se aplica sólo a los miembros. Por lo tanto, no llega a ser universal, en la medida que unas cuantas economías quedan excluidas.

En cuanto al comercio de los bienes, la excepción a la cláusula de nación más favorecida es el artículo XXIV del GATT, dado que permite establecer uniones aduaneras y zonas de libre comercio. Incluso si se establece que los aranceles y las regulaciones no deben ser mayores o más restrictivas que los que se encontraban previamente en vigor, este ha sido un tema controvertido. Por otro lado, la Parte IV añadió a mediados de la década de 1960 beneficiar principalmente a los países en desarrollo. En la década siguiente la cláusula de habilitación también ha sido criticada basada en la incoherencia y la superposición que viola la letra y el espíritu del acuerdo (Panagariya, 1999). Además de los bienes, el artículo V del AGCS exige a los países abstenerse de elevar el nivel global de obstáculos más allá de lo que existía antes del acuerdo. También sobra decir que el corpus de la OMC prevé mecanismos de solución de diferencias entre las partes (Bhagwati, 1999; Panagariya 1999, Serra et al., 1997).

Los intercambios económicos son considerados como fuerzas que impulsan el crecimiento. Por lo tanto, se supone que es un gran incentivo para la mayoría de las economías del mundo para abrir completamente sus mercados, aunque no existan pruebas concluyentes de esto (Rodrik, 1999). Además que 'ceteris paribus', se dice que la apertura de los mercados funciona como una fuerza integradora para las economías. En este sentido, tiende a asociarse con la idea de la integración. Sin embargo, la integración no es sólo un fenómeno económico. Desde la perspectiva actual, no es difícil observar que, en términos históricos, el proteccionismo ha sido generalizado y es una característica de la economía mundial, donde los campeones del liberalismo en 
el extranjero restringen el acceso a algunos de sus mercados específicos y nichos valiosos de mercado (Gilpin, 2001).

Aunado a las preocupaciones del mercado, también ha habido experiencias políticas que impulsan la integración regional con diversas combinaciones de motivos. Tales son los casos de la Unión Europea, el Tratado de Libre Comercio de América del Norte, la Cooperación Económica Asia-Pacífico, etc. Limitar la explicación del regionalismo contemporáneo a las relaciones de intercambio puede ser muy engañoso y proporciona una imagen parcial y determinista. Las actividades económicas no deben ser consideradas de forma aislada. Por el contrario, las actividades económicas son parte de estos procesos, porque están subsumidas en contextos sociales más amplios. A pesar de que son factores de crucial importancia a tener en cuenta, ello no es lo mismo que decir que se puede reducir el análisis de los procesos sociales y políticos a términos puramente económicos, ya que corremos el riesgo de caer en posiciones deterministas.

Entre las razones extra-económicas presentadas por los economistas neo-clásicos ortodoxos para que las economías en desarrollo puedan participar en el comercio internacional y atraer inversión, algunos de los temas relevantes deben apuntar a la seguridad, el poder de las negociaciones, la cooperación y garantizar la permanencia de las reformas (Banco Mundial, 2002a). En la mayoría de los casos la promoción de marcos regulatorios menos intensivos de las actividades económicas, la conversión de las barreras no arancelarias en aranceles decrecientes, la liberalización del comercio y la inversión de flujos, la reducción de las tasas de inflación y la aplicación de las iniciativas de privatización de las empresas propiedad del gobierno, han representado una alineación con el desarrollo económico de la ortodoxia. Además, los ajustes económicos se presentan ahora como incompletos y en la necesidad de ser emparejados con reformas institucionales.

\section{LA CONTINGENCIAS POLÍTICAS DE LOS PROCESOS SOCIALES REGIONALES}

La suposición de que la apertura de una economía está correlacionada con el índice de crecimiento no está respaldada por ninguna prueba concluyente. No sólo existe una débil correlación entre los niveles de las barreras comerciales y el crecimiento a largo plazo, sino que también depende de algunas políticas e instituciones complementarias (Rodrik, 1999). Por otra parte, la preocupación por la equidad no existe, y el abandono sistemático de la función y las capacidades de las acciones sociales hace a la ortodoxia económica neoclásica controversial y poco realista.

Aunque es importante destacar la importancia de la inversión y las políticas macroeconómicas, la afirmación de que cuanto más abierta es una economía mayores las tasas de crecimiento, es contraria a la evidencia empírica de la década perdida del crecimiento económico en América Latina y otras regiones del mundo. No es un secreto que estas inconsistencias han sido 
sistemáticamente ignoradas por los economistas neo-clásicos. Además, la serie de crisis económicas y financieras que han ocurrido desde la década de 1980 han restringido las oportunidades para reestructurar las economías. En particular, ha sido la premisa para la introducción del 'libre mercado' a una nueva orientación, tal como lo defendió originalmente el Consenso de Washington y, actualmente, el pos-Consenso de Washington. La controversia surge porque en muchos casos la imposición o la inducción detallada de recetas económicas a gobiernos legítimos han ido más allá de la consistencia teórica y la evidencia empírica. Sin embargo, no parece existir un vínculo fuerte entre una economía con altas tasas de crecimiento económico y la capacidad para atraer el comercio y los flujos de inversión extranjera directa (Rodrik, 1999).

Sin embargo, dejar de lado las cuestiones de gobernanza y rendición de cuentas por privilegiar supuestos imperativos de mercado es problemático e irresponsable. Una deficiencia clave de los programas de ajuste estructural que a su vez son externamente inducidos y aplicados verticalmente desde los estratos superiores de la sociedad a los inferiores, se encuentra en el hecho de no prestar la debida atención a la participación de los grupos sociales. Dentro de un proceso democrático de toma de decisiones, la decisión de someterse a disciplinas externas corre el riesgo de ser contraproducente, especialmente si los grupos nacionales insatisfechos y las demandas se ven relegadas a fin de satisfacer las demandas de otros objetivos. Someter a las economías a una disciplina de mercado impuesta sin determinar la orientación de las políticas públicas, plantea problemas de gobernabilidad democrática, porque los mercados financieros y los actores tienen poder sobre otras circunscripciones sociales (Rodrik, 1999). Huelga decir que tal curso de acción es antidemocrático e inaceptable, tanto en términos de eficiencia económica y de resultados en la sociedad. Entonces, una aceptación acrítica de axiomas económicos neo-clásicos se transforma en un compromiso interno auto-inducido de prioridad política. Dado que la apertura no garantiza ni el crecimiento ni la equidad, la orientación del desarrollo económico es claramente una decisión política, porque los mercados están subsumidos en contextos sociales y en sistemas políticos más amplios.

Sin menoscabar la importancia de las actividades económicas y los intercambios, está claro que estos no son los únicos factores que explican la segunda ola regional de acuerdos preferenciales de comercio. Para el regionalismo, la proximidad geográfica y la contigüidad son tan importantes como la cohesión de la región. Esto último puede ser entendido en un contexto histórico, social, económico, político, organizativo e incluso militar. Ya que los límites de una región no necesariamente tienen que coincidir con fronteras nacionales, su carácter multidimensional es algo que no debe ser pasado por alto (Fawcett y Hurrell, 1995; Mansfield y Milner, 1997, 1999). Como con la globalización, el regionalismo es un proceso siempre en construcción, en el que las tendencias y contra-tendencias interactúan, dirigidas por agentes situados en su contexto. Antes que enteramente contradictorias, las tendencias globales, transnacionales o nacionales, y el regionalismo son simbióticos y coexisten con otros procesos determinados, tales como las agendas locales, al mismo tiempo que contribuyen a su interminable modificación. Por lo tanto, 
el regionalismo no es un principio de organización para cualquier sistema, ya que constituye sólo uno de los muchos pilares de apoyo para una evolución internacional.

El regionalismo ha sido un proceso originado desde el interior de la sociedad. Se trata de un proceso desde dentro y desde debajo de las regiones en particular, mucho más de lo que se ha reconocido hasta ahora. Esto ha permitido darle un importante rol a los actores participantes, quienes desarrollan sus propias variedades de política social, militar, entorno regulatorio y económico, así como diversas tendencias y contra-tendencias. Una de las razones de este dinamismo se encuentra en la multidimensionalidad de procesos integrales, sociales, políticos, económicos y culturales, formales e informales de redes que se han desarrollado. Aunque en muchos aspectos son importantes, no se encuentran situados necesariamente después de sus propios gobiernos. Considérese el ejemplo de las tendencias de la migración, donde a pesar de los esfuerzos de los gobiernos para detener el flujo de migrantes, hay quienes aspiran a un mayor nivel de vida en un país extranjero. Dichas tendencias son más fuertes y más numerosas que los recursos oficiales para frenar la migración.

Lamentablemente, a pesar de las estrategias discursivas y de marketing, un enfoque integral atento a las condiciones sociales y políticas, no es del tipo que se promueve por las organizaciones internacionales. Tomando en cuenta la influencia que estas organizaciones tienen, las recomendaciones que hacen, los objetivos que apuntan, los métodos que utilizan, y la orientación de sus políticas, en algunos casos tienden a seguir mandatos doctrinarios, en lugar de adoptar sugerencias oportunas. La controversia en torno a la segunda generación de reformas económicas de varios países, que están actualmente en fase o hayan sido objeto de programas de ajuste estructural en el pasado, es de relevancia. Porque su objetivo es hacer hincapié en el papel de las instituciones como herramientas complementarias para los mecanismos de mercado, sin tocar los supuestos clave y métodos irrealistas e inconsistentes. Poniendo atención particular sobre el caso del Banco Mundial, la siguiente sección analiza e ilustra la institucionalidad aparente del 'auto-ajuste' y la 'autorregulación' de los mercados como ejemplo de la influencia del pos-Consenso de Washington.

\section{ADICIÓN DE AXIOMAS: LOS MERCADOS REQUIEREN COMPLEMENTOS INSTI- TUCIONALES}

El Banco Mundial, como una de las más importantes organizaciones internacionales dedicadas a ayudar a los países miembros para alcanzar mayores niveles de desarrollo, ha sido fuertemente criticado por la insuficiencia de sus recomendaciones y la 'talla única' de las recetas a sus países miembros. El enfoque 'favorable al mercado' que esta organización promueve no está exento de problemas. Consideremos, por ejemplo, las estrategias recomendadas al Estado para cumplir ciertas funciones, por un lado, y los consejos para aumentar las capacidades del Estado mediante un nuevo impulso público institucional, por el otro. Se nos dice que la tarea principal del 
Estado es encontrar maneras para reducir las brechas entre las exigencias de sus diferentes grupos y su capacidad de emprender y promover la acción colectiva eficiente (World Bank, 1997). Adoptando la perspectiva de Polanyi (1957), se puede decir que el Banco Mundial recomienda estrategias para que coincida el rol del Estado con sus capacidades, orientado a los procesos de mercantilización; en ese sentido induce ciertos cursos de acción específicos para que los gobiernos funcionen como complemento de los mercados, ya que explícitamente les brinda una receta para la delimitación de las acciones, especificando las omisiones y asesorando sobre las estrategias. Para el Banco Mundial, las principales prioridades de las economías en desarrollo incluyen el logro del mantenimiento de la estabilidad macroeconómica, la atracción de la inversión privada, la coherencia económica entre las políticas gubernamentales, y el objetivo de invertir en infraestructura y servicios sociales. Además, se recomienda el establecimiento del Estado de derecho y la protección de sectores vulnerables de la sociedad y el medio ambiente. Al hacerlo, no sólo da consejos económicos sino, lo que es más importante, añade también una dimensión política más explícita a sus recomendaciones ${ }^{4}$.

A pesar de que el Banco Mundial presta atención a la función y el potencial de los derechos humanos, hay más énfasis en la canalización u orientación de los actores para lograr imperativos económicos. Desde ese punto de vista, la inculcación de los hábitos orientados a la racionalidad de los mercados podría ser considerada como un elemento central de los Estados. Dicho perfil está atento a la institucionalidad, por un lado, pero también al conjunto de estrategias del Estado. Desde la racionalidad económica las necesidades se construyen ya que no son dadas naturalmente. Es también el caso de las actuaciones utilitaristas inducidas, en la que el actor unitario racional legitima la construcción de posiciones filosóficas positivistas y la postura de la ortodoxia económica neoclásica. Reformas institucionales como las realizadas para la implementación de los derechos económicos estructurales y sectoriales de los programas de ajuste, se construyen como componentes vitales del contexto en que los organismos se encuentran. Por otra parte, la modificación en el papel del Estado tiene como objetivo reforzar la creación de la racionalidad económica y la inducción del homo economicus dentro de un entorno institucional (Williams, 1999).

4. Con el fin de alcanzar el objetivo de aumentar la capacidad del Estado para llevar a cabo y entregar eficiencia en la acción colectiva de la revitalización de las instituciones públicas, el Banco Mundial considera que el respeto del Estado de derecho es una condición necesaria pero no suficiente, para alcanzar la meta del desarrollo sostenible. Las reformas políticas recomendadas macro-económicamente se centran en los tipos de cambio, políticas fiscales y comerciales, mientras que las reformas en el ámbito institucional se focalizan en atención a la regulación, los servicios sociales, finanzas e infraestructura (World Bank, 1997).

El Estado debe actuar con un mensaje de "socio, catalizador y facilitador", al mismo tiempo que debe dar nuevo impulso a las instituciones públicas que proporcionan las bases de los mercados, lo que hace que el Banco Mundial adopte una perspectiva estructural de los procesos de cambio social. Deben tomarse en cuenta también particularidades sociales y políticas, dado que los actores colectivos e individuales por igual tienen el potencial de influir, promover y lograr cambios institucionales y estructurales. 
Además de la difusión del punto de vista estructural del Estado que sostiene el Banco Mundial, este pone un fuerte énfasis en el alcance y la importancia de las reformas institucionales. Desde esta perspectiva, no es ninguna sorpresa que el conjunto de normas formales e informales en una sociedad que forman la interacción humana son cruciales para el desarrollo económico y el rendimiento (North, 1990). De acuerdo con el Banco Mundial, el Estado debe hacer coincidir sus capacidades institucionales y sus acciones, si se trata de proporcionar una aplicación coherente de las políticas. Por otra parte, también sostiene que las instituciones y los gobiernos pueden desempeñar un rol en el mejoramiento de las diferencias de conocimientos técnicos e información asimétrica, que es crucial para mejorar el desarrollo y las normas de vida (Banco Mundial, 1998). Se reconoce que las instituciones son importantes como uno de los elementos básicos de un proceso social incluyente que responda a los cambios en las circunstancias cruciales para diversas tareas, tales como la gobernanza, la negociación, la coordinación y la regulación (Banco Mundial, 1999). Destacando no sólo las políticas, sino también los procesos, las instituciones son percibidas por los funcionarios del Banco Mundial como requisitos fundamentales para el logro de un desarrollo económico sostenible (World Bank, 2001). ${ }^{5}$

Sin embargo, por importantes que estos argumentos sean, dejan de lado un aspecto muy importante. Ninguno considera que el rol desempeñado por las acciones sociales es fundamental para el logro de los objetivos económicos, políticos y sociales. Las instituciones no son una condición suficiente para el cambio, ya que necesitan ser procesadas endógenamente por los actores situados en su contexto. Por otra parte, cualquiera que sea el carácter del cambio social, en sus dimensiones económica, política y cultural, debe formar parte de los actores, sean individuales o colectivos, mediante sus participaciones para apropiarse y ser parte activa del proceso (Stiglitz, 1998a, 1998b). Sólo mediante la digestión de forma endógena, la incorporación interna y la reproducción dinámica del cambio, los actores podrán contribuir a las tendencias y contratendencias en curso que aparecen en todos los procesos sociales. Desde una perspectiva foucaultiana se puede argumentar que el poder es inmanente en todas las interacciones sociales y, también, que la capacidad de transformación no es estructuralmente determinada por características institucionales (Foucault, 1977, 1979, 1994). Cuando una transformación cualitativa, política, social y económica es necesaria, el carácter político del poder como un factor esencial del cambio debe tomarse en cuenta.

Influyentes e importantes es la forma como las organizaciones internacionales formulan sus opiniones con justificación de la existencia de ganancia, como suficiente para todas las actividades sociales. Es sin duda importante para las empresas privadas. Sin embargo, cuando se trata

5. La influencia de las instituciones que las organizaciones internacionales muestran en la promoción de su agenda institucional 'favorable al mercado' no pueden ser ignoradas por los países en desarrollo.

El diseño institucional es relevante para las empresas, gobierno y sociedad, y al ser un canal de información sobre las condiciones del mercado, los bienes y los participantes, resulta una función importante para llevar a cabo por el Estado, además de la definición y aplicación de los derechos de propiedad. 
del sector público, tales preocupaciones no son objetivos económicos pertinentes. Según Polanyi $(1957,1992,1996)$, la expansión de los mercados y las actividades económicas crean la necesidad de una garantía y ampliación de la protección social de los mercados, mientras que por otro lado, para Schumpeter $(1934,1954,1996)$, las fluctuaciones cíclicas que fomentan la aparición continua de nuevas formas económicas y sociales nunca son exclusivamente económicas. A diferencia de la mano de obra y las tierras que son naturales, el dinero y conocimientos se producen socialmente, y también se han mercantilizado. De este modo, se incorporan a una forma discursiva inspirada en los supuestos de axiomas mercantilistas asociales y ahistóricos.

Con el beneficio de una visión retrospectiva, después de varias décadas de seguimiento y evaluación, y una gama de diferentes experiencias asociadas a los procesos de reestructuración económica, el Banco Mundial ha tratado de incorporar puntos de vista políticos y sociales en su análisis, como muestra la última serie de sus informes. Por ejemplo, sin degradar la importancia de 'fundamentos económicos', la inclusión social y de puntos de vista políticos para atacar la pobreza, hace hincapié en la promoción de las oportunidades sobre derechos económicos, la facilitación de la capacitación y la mejora de la seguridad social. Este es un tema que no debe posponerse por más tiempo. Sin embargo, el Banco Mundial afirma que el papel de las instituciones y sus interacciones con algunos otros factores sociales y políticos ayudan en la reducción de la vulnerabilidad, la falta de voz, el empoderamiento y la representación en función de los escenarios de la pobreza (World Bank, 2001). El Banco Mundial, haciéndose eco de las afirmaciones de muchos economistas neo-clásicos, ha destacado el lado 'creativo' y la potencialidad de las instituciones como dispositivos útiles para mantener fuertes tasas de crecimiento y, supuestamente, también de desarrollo. Sin embargo, se ha prestado poca atención a los lados 'destructores' de las instituciones, si están mal implementadas y organizadas de forma inadecuada. La debilidad de las instituciones no es neutral con respecto a su entorno social y político, porque tienden a favorecer a determinados sectores y grupos interesados.

Además, la repetición de las prácticas institucionales sobre los pobres no sólo daña a las personas que no se benefician de ningún privilegio, sino que también, debido a su trayectoria dependiente, incrementa el grado de dificultad para romper la inercia y las prácticas dañinas de las reglas formales e informales. En un intento por sacudirse la inercia, el Banco Mundial se comprometió públicamente a trabajar para lograr mutuamente reforzar los Objetivos de Desarrollo del Milenio (ODM), a saber: promoción de la igualdad de género, matrícula escolar de primaria y secundaria y los servicios de salud, reducción de la pobreza, de tasas de mortalidad materna e infantil, y detención de la degradación del medio ambiente para 2015 (World Bank, 2001). Sin embargo, la organización hermana de Bretton Woods, el Fondo Monetario Internacional, se apega más firmemente a los supuestos de 'auto-ajuste' y 'autorregulación' del mercado en su trabajo.

Sin embargo, sería injusto no reconocer que ha habido una evolución en las recomendaciones con respecto a los procesos de desarrollo por parte del Banco Mundial. En un principio, 
el énfasis estaba puesto más en el crecimiento, mientras que hoy en día la amplia agenda se refiere más al crecimiento con equidad. El énfasis en las instituciones se refleja ahora en el pos-Consenso de Washington. Es precisamente la perspectiva institucional presentada y promovida por las organizaciones de Bretton Woods y el Consenso de Washington, que en la siguiente sección de este artículo se tratará mediante una exploración de la primera y segunda generación de reformas económicas en América Latina, según la percepción y su promoción desde Washington.

\section{INSUFICIENCIA DE LAS REFORMAS MACROECONÓMICAS E INSTITUCIONALES}

Las reformas económicas que se han aplicado en América Latina desde principios de 1980 fueron construidas discursivamente como una respuesta a la crisis de la deuda internacional. Los problemas de la deuda internacional fueron considerados por los economistas neoclásicos como dificultades principalmente de insolvencia. Por lo tanto, sus colegas dentro de las organizaciones de Bretton Woods presionaron para los programas de ajuste estructural donde se percibía que la economía había fracasado, adoptando el modelo de la industrialización sustitutiva de importaciones. Dado que los principales bancos en los Estados Unidos eran principalmente afectados por la insolvencia de algunas economías de América Latina, que se encontraban incapaces de reembolsar sus deudas internacionales, la Reserva Federal aplicó presión para evitar daños en el sistema bancario de Estados Unidos y evitar que la crisis se derramara a otros deudores. El uso de un enfoque caso por caso y la renegociación pragmática de reprogramación de la deuda fue seguida por la implementación de diferentes estrategias como los planes Brady y Baker, y la condicionalidad del Fondo Monetario Internacional (FMI). El primer plan, por ejemplo, se centró principalmente en las reformas de ajuste estructural, poniendo énfasis particular en las reformas estrictas con el objetivo de lograr un ajuste en el balance de pagos. La condicionalidad del FMI comenzó a expandirse desde preocupaciones macroeconómicas en las agendas, situación esta que llegaría a ser conocida como el Consenso de Washington y llevaría hacia la segunda generación de reformas económicas.

A pesar de que la promoción de la prosperidad es considerada como el principal objetivo del FMI, al limitarse a los modelos económicos neoclásicos que tienden a concentrarse únicamente en los factores observados, su desempeño ha estado a la altura de sus objetivos y su modus operandi sigue siendo controvertido. Se ha argumentado que la voluntad política y la confianza de los miembros del FMI son factores clave en la selección de candidatos para algún préstamo, así como en la evaluación de su desempeño (Vreeland, 2003). En algunos casos, llegar a un acuerdo económico entre un gobierno y el FMI puede ofrecer una justificación externa para la imposición de reformas económicas internas y para cambiar la censura al Fondo. De esta manera, las políticas impopulares están ligadas a condicionantes externos, a aumentar los grados de apalancamiento y el rechazo al aumento de los costos de la población nacional. Dado que la responsabilidad de la aplicación de los programas de ajuste económico corresponde a un 
gobierno que llega a un acuerdo con el FMI, siempre hay margen de maniobra para decidir sobre las políticas a adoptar, con qué gravedad y cuándo (Vreeland, 2003). Así, el papel del organismo sigue siendo crucial, a pesar de argumentos en sentido contrario.

Los programas del FMI desalientan el crecimiento, empeoran la desigualdad y mantienen un registro mixto en la promoción de la estabilidad. La aplicación de sus acuerdos por número de países muestra los patrones de desaceleración del crecimiento económico y cada vez más la redistribución equitativa del ingreso, beneficiando a los más ricos a expensas de los que están en peor situación (Vreeland, 2003). Por lo general, la estabilización económica y los programas de ajuste, tal como fueron diseñados por el FMI, se han unido históricamente a los préstamos de diferentes condicionalidades. Además, está el hecho de que el FMI sólo alcanza acuerdos con los Ministerios de Finanzas de los países miembros y que no pasa por la aprobación de otras ramas del gobierno, ya que no se requiere la ratificación legislativa (Vreeland, 2003). Sin embargo, en los acuerdos con el FMI, los cambios de política necesarios se realizan ex post y los recursos que se prestan a los países miembros se ponen a disposición en el transcurso del contrato, pero solo si los cambios de política observados se consideran suficientes y satisfactorios. El pragmatismo de estos mecanismos significa que la ratificación o la aprobación de otras ramas del gobierno, si son necesarios para los cambios de políticas específicas establecidas en el acuerdo, no tienen por qué ser de preocupación para el FMI. Es solo el poder Ejecutivo del país el que tiene que hacer frente a ello (Vreeland, 2003).

El Consenso de Washington se ha asociado con la agenda ideológica del neoliberalismo, tal y como señalaran economistas como Friedman y Hayek, junto con los programas económicos implementados durante la década de 1980 y asociados con la ex Primera Ministra Thatcher del Reino Unido y el ex Presidente Reagan de los Estados Unidos. La etiqueta fue pensada originalmente para describir un conjunto de reformas políticas e instrumentos económicos que alcanzaron un cierto grado de acuerdo entre los funcionarios del Congreso de Estados Unidos, instituciones financieras internacionales como el FMI, el Banco Mundial y el Banco Interamericano de Desarrollo, las agencias económicas del gobierno de Estados Unidos, la Reserva Federal y centros de investigación (Williamson, 1990). El objetivo principal del Consenso de Washington fue la reactivación y promoción del crecimiento económico en América Latina, objetivo que, sin embargo, se opone a cualquier preocupación por la redistribución. De hecho, los instrumentos de política consideran: (a) la eliminación de los déficits fiscales; (b) la reorientación del gasto público para la educación primaria, la salud e infraestructura; (c) la implementación de la base imponible; (d) las tasas de interés positivas pero moderadas; (e) el uso del tipo de cambio competitivos para inducir el crecimiento de las exportaciones no tradicionales; (f) la sustitución progresiva de las restricciones comerciales cualitativas disminuyendo aranceles; (g) la abolición de barreras de la IED; (h) la privatización de empresas estatales; (i) la liberalización de la entrada de empresas y la competencia; y (j) la provisión de derechos de propiedad seguros (Williamson, 1990). Dicho conjunto de políticas económicas se convirtió en una directriz a llevar a cabo para los gobiernos de la región latinoamericana. 
En la práctica, algunos puntos como la liberalización financiera se llevaron a cabo con entusiasmo, mientras que otros, como la reforma tributaria fue inferior a las aspiraciones origina$l_{\text {les}}^{6}$. Sin embargo, los resultados globales de las economías de América Latina después de años de implementación de la reestructuración económica siguen siendo muy decepcionantes, incluso hoy en día. No sólo los índices deseados de crecimiento económico no se han alcanzado, sino que incluso tasas negativas han aparecido en algunos años desde la década de 1980. A pesar de reconocer que las circunstancias adversas, tales como terremotos y huracanes, que están más allá de cualquier control humano, habían afectado a la región en su conjunto y en algunos países en lo individual, la promesa de un crecimiento sostenido aún no se cumplió, incluso para las economías en mejor situación durante los períodos relativamente mejores. Por otra parte, una consecuencia no intencional parece haber sido la concentración del ingreso, lo que ha hecho a la región en general, y en algunos países en particular, muy polarizados.

Las expectativas iniciales de los diez puntos del Consenso de Washington son que los flujos de capital y el crecimiento de las exportaciones promoverían el desarrollo en sectores de trabajo intensivo. Como estas expectativas se quedaron cortas en realidad, el diseño institucional deficiente fue señalado como el principal culpable. Analistas han empujado una creciente demanda de reformas institucionales en América Latina y el Caribe como respuesta a las tendencias de la integración financiera. Las instituciones más fuertes y mejores se consideran críticas para la estabilidad macroeconómica. Tal enfoque tiene como objetivo reforzar y mantener los ajustes macro-económicos que la mayoría de los países de la región han vivido durante las últimas décadas. En consecuencia, se afirma que las instituciones son importantes porque constituyen un conjunto de "... reglas formales e informales, y sus mecanismos de cumplimiento moldean el comportamiento de los individuos y las organizaciones de la sociedad..." (Burki y Perry, 1998: 11). Su relevancia por el bien de la estabilidad se pone de relieve. Sin embargo, la estabilidad, a su vez, se percibe como seriamente amenazada por problemas técnicos y políticos de la región, donde los problemas de información y su aplicación son importantes. El pos-Consenso de Washington no es más que la promoción de la convergencia legal, técnica y política, donde las leyes, reglamentos, constituciones y contratos formales estarían de acuerdo con las prácticas informales basados en la confianza, la ética, los valores y las normas políticas.

6. El asesoramiento a los mercados de libre 'represión financiera' se supone que funciona en las economías imperfectas en los mercados de capital, mediante el aumento de la demanda de dinero, lo que ayuda a expandir el sistema de política monetaria (McKinnon, 1973). Directrices amplias y generales para el orden óptimo de la liberalización económica se pusieron en práctica en los mercados emergentes durante las dos décadas finales del siglo pasado (McKinnon, 1991). Sin embargo, en algunos casos, la implementación de políticas imprudentes condujeron al fracaso y espirales de crisis. Hoy en día, incluso los defensores entusiastas de la idea de la liberalización de los mercados de capitales en las economías en desarrollo de cualquier 'represión financiera', han reconocido posteriormente el hecho de que las regulaciones financieras deben ser observadas por parte de las economías en la actualidad sobre el 'juego de patrón del dólar' (McKinnon, 2002). 
Como resultado de ello, todas estas instituciones formales e informales se suponen aptas para determinar la eficacia y la existencia de ambos mercados y sus organizaciones. Independientemente de su carácter público o privado, se espera proporcionar información clara, que las reglas sean ampliamente conocidas, coherentes, universales, previsibles y creíbles, correcta y uniformemente aplicadas (Burki y Perry, 1998). Las recomendaciones no se detienen ahí. Se sugiere incluso potenciar los beneficiarios de las reformas institucionales y la compensación para los perdedores, brindando incluso apoyo político para futuras reformas y proporcionar incentivos para mantenerlas en marcha. Una gran visión de certeza predecible es muy poco realista, ya que insiste sobre los modos de inferencia deductiva y positivista, de sistemas cerrados que no corresponden con la realidad, como se argumenta en este artículo. Las explicaciones ortodoxas neoclásicas por los malos resultados económicos en los países donde se puso la primera generación de reformas económicas en la práctica tienden a centrarse en una aplicación incorrecta e incompleta. Desde la misma perspectiva, también se argumenta que el énfasis original, principalmente en el crecimiento económico, era demasiado estrecho y, por tanto, debe ser complementado para completar el desarrollo económico, la reestructuración y la aplicación de una segunda generación de reformas institucionales. Junto con estos paquetes, la nueva agenda incluye disposiciones para reducir la probabilidad de los problemas de equidad y crisis social (Kuczynski y Williamson, 2003). Las reformas que se espera que sean completadas incluyen la desregulación del trabajo y los mercados de productos, nuevas reformas del comercio y la privatización de las empresas que son propiedad del Estado; la reducción de los mercados informales y el acceso a los mercados desarrollados a través de la Organización Mundial del Comercio (OMC), y acuerdos regionales como el Tratado de Libre Comercio de América del Norte (Cuadra Montiel, 2008). Para este punto de vista neoclásico ortodoxo, la construcción de la estructura institucional de la reforma incluye la modernización del sistema político, la administración pública, el poder judicial y el sector financiero.

A pesar de que no se puede argumentar que ha habido un cambio dramático en la economía actual, el énfasis en las instituciones se presenta como un intento de ampliar la estrecha preocupación por el crecimiento económico. Es evidente que la equidad nunca fue una prioridad para el Consenso de Washington (Kuczynki y Williamson, 2003); a pesar de un cierto reconocimiento de la importancia de las instituciones para lograr el crecimiento y la equidad, parece que las iniciativas y las propuestas no son suficientemente fuertes para lograr el desarrollo sostenible y costos razonables de crecimiento, sin tener en cuenta la integración social de los mercados y transacciones económicas. A pesar de que tiene una de las distribuciones más desiguales de ingresos y activos del mundo, el objetivo de reducir la pobreza y mejorar la equidad sin sacrificar el crecimiento en América Latina se trata ahora en las organizaciones internacionales, organismos gubernamentales y la academia, como un nuevo objetivo primordial. Para la mayoría de esas economías, las décadas de 1980 y 1990 fue un período de reestructuración económica y de resultados decepcionantes, sobre todo con respecto a las condiciones de crecimiento económico, reducción de la pobreza, la distribución del ingreso social. El desafío de construir sociedades más eficientes, que no justas, sólo a través de una mezcla de instrumentos económicos, 
es uno al que no pocos de los analistas canalizan sus esfuerzos profesionales ${ }^{7}$. No sólo ha sido reconocido que la pobreza y la desigualdad actúan como elementos disuasorios para el crecimiento, sino que también fortalecen y refuerzan patrones no deseados de polarización y miseria. Para la segunda generación de reformas económicas, algunas de las herramientas institucionales para la equidad social económica que ahora se promueven, es el énfasis en la eficiencia económica, como mantener la disciplina fiscal, reducir los subsidios a los ricos para la educación superior y la reducción de la evasión de impuestos. Otras preocupaciones incluyen mantener un ojo en las cuestiones de equidad, tales como más gasto público en redes de seguridad social, protección de los derechos de los trabajadores y la lucha contra las prácticas sobre la discriminación (Birdsall y De la Torre, 2001).

Sin embargo, los problemas que están fuera del alcance de la economía nacional e institucional son las reformas económicas sobre las barreras al comercio de productos básicos y bienes manufacturados de países en desarrollo, como las encontradas en los mercados más grandes y más rentables del mundo. Por ejemplo, el proteccionismo contra importaciones de textiles y productos agrícolas en los Estados Unidos y en la Unión Europea tiende a agravar los patrones de pobreza, lo que refuerza la desigualdad en los países cuyos principales productos de exportación no pueden beneficiarse del acceso a los mercados desarrollados. Por lo tanto, la reducción o, preferentemente, la eliminación de dichos obstáculos en los mercados de la Organización para la Cooperación y el Desarrollo Económico (OCDE), desplazarían la producción hacia los productores de menor costo en economías en desarrollo y constituirían un paso hacia una situación más equitativa en estos países (Birdsall y De la Torre, 2001). Estas exigencias no son nuevas. Han estado en innumerables mesas de negociación, y, aún hoy, continúan sin resolverse, lo que por tanto representa un tema sensible en las negociaciones comerciales entre los Estados Unidos y la Unión Europea. Particularmente importante en este sentido han sido los subsidios y el proteccionismo en sectores de servicios intensivos en mano de obra como la agricultura y el acero. No es ningún secreto que el acceso al mercado es crucial para la orientación hacia el exterior de prácticas e instituciones económicas en los países en desarrollo.

El problema es que la agenda de reformas institucionales están mal ejecutadas o puestas en práctica en una forma que refuerce los patrones existentes favorables a la desigualdad (CuadraMontiel, 2009). Para decirlo de manera concisa, el peligro es que el objetivo de crecimiento sostenible y equitativo no se alcance. La constante repetición de las prácticas de comercialización sólo puede llevar aun más a la mercantilización, sin importar el cambio en el discurso y la retórica. Si las prácticas económicas, las condiciones y las instituciones han cambiado y orientado a servir a fines más equitativos, esto solo podría ser considerado como un paso preliminar. Por ejemplo, la implementación de las estrategias económicas y el uso orientado hacia el

7. Cfr. Birdsall y De la Torre (2001); Burki y Perry (1998); Kuczynski y Williamson (2003); y los Informes del Banco Mundial de los últimos años, entre otros. 
exterior de las políticas económicas han favorecido la polarización, la desvinculación de la oferta interna de insumos para exportar productos manufacturados en el México contemporáneo y en algunos otros países de América Latina. Por otra parte, ya que un puñado de empresas representa una parte significativa de los flujos hacia el norte y hacia el sur de la frontera con Estados Unidos de los productos, su impacto general en el mercado laboral es muy bajo (Dussel Peters, 1997, 2000). Si la segunda generación de reformas económicas ha de tener éxito, un mucho más fuerte y más decisivo énfasis en el mercado interno no debe retrasarse, sobre todo si ha de ofrecer condiciones de crecimiento endógeno (Cuadra-Montiel, 2012). El riesgo de poner demasiado énfasis en la flexibilidad laboral y de metas de inflación es que esto no crea puestos de trabajo de alta calidad según sean necesarios, ni es probable que lo hagan en el futuro tampoco. En efecto, un número insuficiente de nuevos empleos con crecimiento salarial negativo en términos reales no puede entregar crecimiento equitativo sostenible endógeno.

\section{CONCLUSIONES}

Dado que los procesos asociados con la globalización son a todas luces no por el mercado, es necesario reconocer el carácter altamente político de compulsiones presuntamente económicas. La convicción de que el discurso político de la globalización es una homogeneización de fuerzas económicas impulsadas también se puede encontrar en el modelo de equilibrio general. $\mathrm{Ni}$ la globalización ni el discurso del modelo de equilibrio general reconocen las diferencias institucionales y los determinantes sociales de los mercados, ya que se presentan como homogéneos y convergentes. Con base en estos supuestos no realistas, sin embargo, las propiedades del sistema cerrado del modelo se utilizan para prescribir cursos de acción política y económica. El problema de la inaplicabilidad de los modelos de orientación matemática ortodoxa neoclásica radica en la falta de correspondencia entre la teoría y la práctica, los supuestos no realistas, su ontología positivista y sus inferencias deductivistas. Tienden a ser asociales, ahistóricas, reduccionistas y deterministas, al mismo tiempo que descartan la importancia de los actores para inyectar intencionalidad, indeterminación e incertidumbre a los procesos.

La concentración de los flujos de comercio e inversión entre Europa Occidental, Asia Pacífico y América del Norte, junto a sus acuerdos regionales, ofrecen una imagen más diferenciada y más compleja de lo que supuestamente las fuerzas de la homogeneización del mercado hacen. Diferentes escalas espaciales, tales como mundial, regional, nacional y local no son incompatibles entre sí, ni lo son con el significado de los actores en la conformación de las condiciones que les rodean. En este sentido, instituciones internacionales como el GATT/OMC reflejan este tipo de situaciones en sus marcos normativos y operativos. Además, los bloques regionales tienen fuertes rasgos políticos que son extraeconómicos e incluyen, pero no se limitan a, la seguridad, el poder de negociación, la cooperación y garantizar la permanencia de las reformas. No sólo son los procesos políticos regionales y contingentes; también se originan dentro de la sociedad. Originalmente empiezan desde dentro y desde abajo de las regiones, en particular. 
Como se señaló anteriormente, no hay evidencia concluyente de que el grado de apertura de una economía esté correlacionado con un fuerte ritmo de crecimiento. Sin embargo, este último tiende a funcionar como imán para atraer el comercio y los flujos de inversión. Para muchos países, la aceptación acrítica de los axiomas neo-clásicos se ha transformado en prioridades políticas nacionales auto-inducidas. Mediante la promoción de grados crecientes de apertura en las economías a través de los flujos de capital y de promoción de exportaciones, y por no estar al tanto de las cuestiones de equidad, el Consenso de Washington ha fracasado en cumplir con la promesa de una mayor tasa de crecimiento. Aun cuando el énfasis en la liberalización financiera, junto con el resto de la primera generación de reformas económicas estaba en su lugar, sin que sus resultados coincidieran con las expectativas de crecimiento económico, el pos-Consenso de Washington se centró más en las reformas institucionales.

La segunda generación de reformas tiene como objetivo corregir la inadecuada e incompleta implementación de la reestructuración económica mediante la reforma de los marcos institucionales de los países en desarrollo. Las estrategias del pos-Consenso de Washington incluyen modernización de sus sistemas políticos nacionales, junto con características de su servicio civil, la judicatura y su sector financiero. Contempla también provisiones para reducir la probabilidad de problemas de equidad social y la crisis. El pos-Consenso de Washington considera entre otros aspectos estrategias de la reducción de los marcos regulatorios de los mercados laborales y de mercancías y servicios, una mayor liberalización comercial, privatización de programas, reducción de los mercados informales, y cada vez mayor acceso a los mercados desarrollados. La importancia de las instituciones para realizar tareas tales como la gobernabilidad, la negociación, la coordinación y la regulación se han destacado como elementos cruciales de los procesos sociales capaces de hacer frente a las circunstancias cambiantes. Sin embargo, las instituciones no están en condiciones de garantizar ningún resultado específico en absoluto, a menos que sean considerados como una herramienta que los actores situados en su contexto podrían emplear. Las reformas institucionales jamás son neutrales, ya que muestran un fuerte carácter político que puede tener efectos positivos y negativos. En lugar de ser consideradas como un objetivo, son el medio en el que los actores pueden confiar.

\section{REFERENCIAS}

- $\quad$ Bhagwati, J. (1999). Regionalism and Multilateralism: An Overview. In J. Bhagwati, P. Krishna and A. Panagariya (eds.), Trading Blocs: Alternative Approaches to Analyzing Preferential Trade Agreements (pp. 3-32). London: MIT Press.

- $\quad$ Bhagwati, J. and A. Panagariya (1999). Preferential Trading Areas and MultilateralismStrangers, Friends or Foes? In J. Bhagwati, P. Krishna and A. Panagariya (eds.), Trading Blocs: Alternative Approaches to Analyzing Preferential Trade Agreements (pp. 33-100). London: MIT Press. 
- Bhagwati, J., Greenaway, D. and Panagariya, A. (1998). Trading Preferentially: Theory and Policy. The Economic Journal, 108, 1128-1148.

- $\quad$ Birdsall, N. and De La Torre, A. (2001). Washington Contentious: Economic Policies for Social Equity in Latin America. Washington DC: Carnegie Endowment for International Peace and the Inter-American Dialogue.

- $\quad$ Burki, S. J. and Perry, G. (1998). Beyond the Washington Consensus: Institutions Matter. Washington DC: The World Bank.

- Cuadra-Montiel, H. (2012). Globalization and Re-Commodification in México. In: H. Cuadra-Montiel (ed.), Globalization: Approaches to Diversity (pp. 185-222). Croatia: InTech.

- Cuadra-Montiel, H. (2011). Demystifying Globalization and the State: Preliminary Comments on Re-Commodification, Institutions and Innovation. In: P. Pachura (ed.), The Systemic Dimension of Globalization (pp. 83-108). Croatia: InTech.

- $\quad$ Cuadra-Montiel, H. (2009). Social Change in Mexico: Re-Politicizing the Agenda of Reform. International Review of Sociology - Revue Internationale de Sociologie, 19 (3), 411-432.

- $\quad$ Cuadra-Montiel, H. (2008). Where Does Mexico Stand? Interpreting NAFTA's Regional Scope and the FTAA Hemispheric Project. Norteamérica, 3 (1), 65-93.

- Cuadra-Montiel, H. (2007). Critical Realism and the Strategic Relational Approach: Comments on a Non KWNS-SWPR Experience. Journal of Critical Realism, 6 (1), 84-110.

- Dussel Peters, E. (1997). La Economía de la Polarización: Teoría y Evolución del Cambio Estructural de las Manufacturas Mexicanas (1988-1996). México: UNAM and Editorial Jus.

- $\quad$ Dussel Peters, E. (2000). Polarizing Mexico: The Impact of Liberalization Strategy. London: Lynne Rienner.

- $\quad$ Fawcett, L. and Hurrell, A. (eds.) (1995). Regionalism in World Politics: Regional Organization and International Order. Oxford: Oxford University Press.

- $\quad$ Foucault, M. (1994). Two Lectures. In M. Kelly (ed.), Critique and Power: Recasting the Foucault/Habermas Debate (pp. 17-46). Cambridge: MIT Press.

- Foucault, M. (1977). Discipline and Punish: The Birth of the Prision. London: Penguin.

- $\quad$ Foucault, M. (1979). The History of Sexuality: An Introduction (Vol. 1) (Translated by R. Hurley). Harmondsworth: Penguin. 
- Gilpin, R. (2001). Global Political Economy: Understanding the International Economic Order. Princeton: Princeton University Press.

- Granovetter, M. (1985). Economic Action and Social Structure: The Problem of Embeddedness. American Journal of Sociology, 91 (3), 481-510.

- Granovetter, M. (1992). Economic Institutions as Social Constrictions: A Framework for Analysis. Acta Sociologica, 35 (3), 3-11.

- Granovetter, M. and Swedberg, R. (eds.) (1992). The Sociology of Economic Life. Colorado: Westview.

- Hay, C. (1996). Re-stating Social and Political Change. Buckingham: Open University.

- Hay, C. (2001). The Invocation of External Economic Constraint: A Genealogy of the Concept of Globalization in the Political Economy of the British Labour Party, 1973-2000. The European Legacy, 6 (2), 233-249.

- Hay, C. (2002). Political Analysis: A Critical Introduction. Hampshire: Palgrave.

- Hay, C. and Marsh, D. (eds.) (2000). Demystifying Globalization. Hampshire: Palgrave.

- Held, D., MCGrew, A., Goldblatt, D. and Perraton, J. (1999). Global Transformations: Politics, Economics and Culture. Cambridge: Polity.

- Jessop, B. (1990). State Theory: Putting the Capitalist State in its Place. Cambridge: Polity.

- $\quad$ Kuczynski, P. P. and Williamson, J. (eds.) (2003). After the Washington Consensus: Restarting Growth and Reform in Latin America. Washington DC: Institute for International Economics.

- $\quad$ Lawson, T. (1997). Economics and Reality. London: Routledge.

- $\quad$ Lawson, T. (2003). Reorienting Economics. London: Routledge.

- Mansfield, E. D. and Milner, H. V. (eds.) (1997). The Political Economy of Regionalism. New York: Columbia University Press.

- Mansfield, E. D. and Milner, H. V. (eds.) (1999). The New Wave of Regionalism. International Organization, 53 (3), 589-627.

- Mckinnon, R. (1973). Money and Capital in Economic Development. Washington DC: Brookings. 
- Mckinnon, R. (1991). The Order of Economic Liberalization: Financial Control in the Transition to a Market Economy. Baltimore: Johns Hopkins.

- Mckinnon, R. (2002). The World Dollar Standard and Emerging Markets. Paper presented at the MaxwellFry Global Finance Lecture. The University of Birmingham. October.

- North, D. C. (1990). Institutions, Institutional Change and Economic Performance. Cambridge: Cambridge University Press.

- $\quad$ Ohmae, K. (1990). The Borderless World: Power and Strategy in the Interlinked Economy. London: Collins.

- $\quad$ Ohmae, K. (1996). The End of the Nation State: The Rise of Regional Economies. London: Harper Collins (Special overseas edition).

- $\quad$ Panagariya, A. (1999). Regionalism in Trade Policy: Essays on Preferential Trading. Singapore: World Scientific.

- $\quad$ Polanyi, K. (1957). The Great Transformation: The Political and Economic Origins of Our Time. Boston: Beacon.

- $\quad$ Polanyi, K. (1992). The Economy as Instituted Process. In M. Granovetter and R. Swedberg (eds.), The Sociology of Economic Life (pp. 29-51). Colorado: Westview.

- $\quad$ Polanyi, K. (1996). Our Obsolete Market Mentality: Civilization Must Find a New Thought Pattern. In R. Swedberg (ed.), Economic Sociology (pp. 109-154). Cheltenham: Elgar.

- $\quad$ Rodrik, D. (1999). The New Global Economy and Developing Countries: Making Openness Work. Washington DC: Overseas Developing Council.

- $\quad$ Schumpeter, J. A. (1934). The Theory of Economic Development: An Inquiry into Profits, Capital, Credit, Interest and the Business Cycle. Cambridge: Harvard University Press.

- $\quad$ Schumpeter, J. A. (1954). Capitalism, Socialism and Democracy (Fourth edition). London: Unwin.

- $\quad$ Schumpeter, J. A. (1996). The Crisis of the Tax State. In R. Swedberg (ed.), Economic Sociology. (pp. 81-114). Cheltenham: Elgar.

- $\quad$ Serra, J., et al. (1997). Reflections on Regionalism: Report of the Study Group on International Trade. Washington DC: Carnegie Endowment for International Peace. 
- $\quad$ Smelser, N. J. and Swedberg, R. (1994). The Sociology Perspective on the Economy. In N. J. Smelser and R. Swedberg (eds), The Handbook of Economic Sociology (pp. 1-26). Princeton: Princeton and Russel Sage Foundation.

- $\quad$ Stiglitz, J. E. (1998a). More Instruments and Broader Goals: Moving Toward the Post-Washington Consensus. The 1998 WIDER Annual Lecture. Helsinki. January.

- $\quad$ Stiglitz, J. E. (1998b). Redifining the Role of the State: What Should it Do? How Should it Do it? And How Should these Decisions be Made? Paper presented at the 10th Anniversary of MITI Research Institute (Tokyo, Japan), March 1998.

- $\quad$ Strange, S. (1994). States and Markets (Second edition). London: Pinter.

- $\quad$ Strange, S. (1996). The Retreat of the State: The Difussion of Power in the World Economy. Cambridge: Cambridge University Press.

- $\quad$ Swedberg, R. (1991). Major Traditions in Economic Sociology. Annual Review of Sociology, $17,251-276$.

- $\quad$ Swedberg, R. (1994). Markets as Social Structures. In N. J. Smelser and R. Swedberg (eds), The Handbook of Economic Sociology (pp. 225-282). Princeton: Princeton and Russel Sage Foundation.

- Vreeland, J. R. (2003). The IMF and Economic Development. Cambridge: Cambridge University Press.

- Watson, M. (2003). Constructing and Contesting Orthodoxies: General Equilibrium Economics and the Poltical Discourse of Globalization. Paper presented at the Annual Conference of the International Studies Association. Oregon. February.

- Williams, D. (1999). Constructing the Economic Space: The World Bank and the Making of the Homo Oeconomicus. Millennium: Journal of International Studies, 28 (1), 79-99.

- Williamson, J. (1990). What Washington Means by Policy Reform. In J. Williamson (ed.), Latin American Adjustment: How Much Has Happened? (pp. 7-20). Washington DC: Institute for International Economics.

- World Bank (1997). World Development Report 1997. The State in a Changing World. Washington DC: The World Bank and Oxford.

- $\quad$ World Bank (1998). World Development Report 1998/1999. Knowledge for Development. New York: The World Bank and Oxford. 
- World Bank (1999). World Development Report 1999/2000. Entering the 21st Century. New York: The World Bank and Oxford.

- World Bank (2000a). Trade Blocs: A World Bank Policy Research Report. New York: The World Bank and Oxford.

- World Bank (2000b). World Development Report 2000/2001. Attacking Poverty. New York: The World Bank and Oxford.

- World Bank (2001). World Development Report 2002. Building Institutions for Markets. New York: The World Bank and Oxford. 